\title{
NODAL NONCOMMUTATIVE JORDAN ALGEBRAS AND SIMPLE LIE ALGEBRAS OF CHARACTERISTIC $p$
}

\author{
BY \\ R. D. SCHAFER(1)
}

A noncommutative Jordan algebra $A$ over a field $F$ is a nodal algebra [9] $\left.{ }^{2}\right)$ in case every element of $A$ may be written in the form $\alpha 1+z$ where $\alpha$ is in $F, 1$ is the unity element of $A$, and $z$ is nilpotent, while the set $N$ of nilpotent elements is not a subalgebra of $A . F$ is necessarily of characteristic $p>0$.

L. A. Kokoris gave in [5] the first examples of simple nodal noncommutative Jordan algebras. Generalizing these, he has constructed in [7] a class $K$ of nodal noncommutative Jordan algebras $A$ of dimension $p^{n}$, and has proved $[6 ; 7]$ that each simple nodal noncommutative Jordan algebra of characteristic $p \neq 2$ is in $K$. Although not all of the algebras in $K$ are simple, it turns out that those algebras with which we are principally concerned here are indeed simple.

Any algebra $A$ of dimension $p^{n}$ in $K$ may be represented as follows: let $B_{n}=F\left[x_{1}, \cdots, x_{n}\right], x_{i}^{p}=0$, be a truncated polynomial ring with partial differentiation operators $\partial / \partial x_{i}$. Write $f \cdot g$ for the commutative associative product in $B_{n}$. Then $A$ is the same vector space as $B_{n}$, but multiplication in $A$ is defined by

$$
f g=f \cdot g+\sum_{i, j=1}^{n} \frac{\partial f}{\partial x_{i}} \cdot \frac{\partial \sigma}{\partial x_{j}} \cdot c_{i j}, \quad c_{i j}=-c_{j i},
$$

where the $c_{i j}\left(=-c_{j i}\right.$ ) are elements in $B_{n}$ which are arbitrary except for the proviso that at least one of them has an inverse (equivalently, at least one of them is not in the radical $N$ of $B_{n}$ ). Then necessarily $n \geqq 2$. Also we note that

$$
c_{i j}=\frac{1}{2}\left[x_{i}, x_{i}\right], \quad i, j=1,2, \cdots, n .
$$

This representation of an algebra $A$ in $K$ is not unique. It is well-known [4, p. 108 ; or $8, \S 4.5$, Proposition 6$]$ that any representatives in $B_{n}$ of the elements of any basis of the $n$-dimensional space $N / N \cdot N$ will serve for the

Presented to the Society, January 20, 1959; received by the editors February 13, 1959.

(1) National Science Foundation Senior Postdoctoral Fellow.

(2) Numbers in brackets refer to the references cited at the end of the paper. 
$x_{i}(i=1, \cdots, n)$. By the chain rule $\partial f / \partial x_{i}=\sum_{k=1}^{n}\left(\partial f / \partial y_{k}\right) \cdot\left(\partial y_{k} / \partial x_{i}\right)$, one obtains

$$
f g=f \cdot g+\sum_{k, l=1}^{n} \frac{\partial f}{\partial y_{k}} \cdot \frac{\partial g}{\partial y_{l}} \cdot d_{k l}
$$

with

$$
d_{k l}=\frac{1}{2}\left[y_{k}, y_{l}\right]=\sum_{i, j=1}^{n} \frac{\partial y_{k}}{\partial x_{i}} \cdot \frac{\partial y_{l}}{\partial x_{j}} \cdot c_{i j},
$$

where $y_{1}, \cdots, y_{n}$ are any elements of $B_{n}$ whose residue classes form a basis for $N / N \cdot N$ over $F$.

In this paper we study the derivations of the algebras in $K$. We are led to relationships between some of these algebras and recently discovered simple Lie algebras of characteristic $p$. In particular, we obtain an intrinsic characterization of the simple Lie algebras $V_{r}$ of A. A. Albert and M. S. Frank [1]. Also we display each of the simple Lie algebras $L(G, \delta, f)$ of characteristic $\neq 2$ defined by Richard Block [2] as an ideal in the derivation algebra of a suitably chosen simple nodal noncommutative Jordan algebra $A$. We assume characteristic $p>2$ throughout.

1. Derivations. Since $c_{i j}=-c_{j i}$ in (1), we have $(f g+g f) / 2=f \cdot g$. That is, the commutative algebra $A^{+}$attached to $A$ is $B_{n}$ itself.

Clearly any derivation of $A$ is also a derivation of $A^{+}$. But the derivations of $B_{n}$ are well-known [4, p. 107]:

$$
f \rightarrow \sum_{k=1}^{n} \frac{\partial f}{\partial x_{k}} \cdot a_{k}
$$

for arbitrary elements $a_{k}$ in $B_{n}$. Hence, if $D$ is a derivation of $A$, we have

$$
f D=\sum_{k=1}^{n} \frac{\partial f}{\partial x_{k}} \cdot a_{k}
$$

for all $f$ in $A$ and for certain elements $a_{k}\left(=x_{k} D\right)$ in $A(k=1, \cdots, n)$. Occasionally we shall employ the notation $D=\left(a_{1}, \cdots, a_{n}\right)$ used in [3] and [1].

Suppose that $D$ is given by (4). Then $D$ is a derivation of $A^{+}$, and also

$$
\begin{aligned}
\frac{\partial h}{\partial x_{i}} D-\frac{\partial(h D)}{\partial x_{i}} & =\sum_{k}\left(\frac{\partial^{2} h}{\partial x_{k} \partial x_{i}} \cdot a_{k}-\frac{\partial^{2} h}{\partial x_{i} \partial x_{k}} \cdot a_{k}-\frac{\partial h}{\partial x_{k}} \cdot \frac{\partial a_{k}}{\partial x_{i}}\right) \\
& =-\sum_{k} \frac{\partial h}{\partial x_{k}} \cdot \frac{\partial a_{k}}{\partial x_{i}}
\end{aligned}
$$

for any $h$ in $A$. Hence 


$$
\begin{aligned}
(f g) D- & (f D) g-f(g D)=(f \cdot g) D-(f D) \cdot g-f \cdot(g D) \\
& +\sum_{i, j}\left\{\left(\frac{\partial f}{\partial x_{i}} \cdot \frac{\partial g}{\partial x_{j}} \cdot c_{i j}\right) D-\frac{\partial(f D)}{\partial x_{i}} \cdot \frac{\partial g}{\partial x_{j}} \cdot c_{i j}-\frac{\partial f}{\partial x_{i}} \cdot \frac{\partial(g D)}{\partial x_{j}} \cdot c_{i j}\right\} \\
= & \sum_{i, j}\left\{\frac{\partial f}{\partial x_{i}} D \cdot \frac{\partial g}{\partial x_{j}} \cdot c_{i j}+\frac{\partial f}{\partial x_{i}} \cdot \frac{\partial g}{\partial x_{j}} D \cdot c_{i j}+\frac{\partial f}{\partial x_{i}} \cdot \frac{\partial g}{\partial x_{j}} \cdot c_{i j} D\right. \\
& \left.\quad-\frac{\partial(f D)}{\partial x_{i}} \cdot \frac{\partial g}{\partial x_{j}} \cdot c_{i j}-\frac{\partial f}{\partial x_{i}} \cdot \frac{\partial(g D)}{\partial x_{j}} \cdot c_{i j}\right\} \\
= & \sum_{i, j, k}\left(-\frac{\partial f}{\partial x_{k}} \cdot \frac{\partial a_{k}}{\partial x_{i}} \cdot \frac{\partial g}{\partial x_{j}} \cdot c_{i j}-\frac{\partial f}{\partial x_{i}} \cdot \frac{\partial g}{\partial x_{k}} \cdot \frac{\partial a_{k}}{\partial x_{j}} \cdot c_{i j}+\frac{\partial f}{\partial x_{i}} \cdot \frac{\partial g}{\partial x_{j}} \cdot \frac{\partial c_{i j}}{\partial x_{k}} \cdot a_{k}\right) \\
= & \sum_{i, j} \frac{\partial f}{\partial x_{i}} \cdot \frac{\partial g}{\partial x_{j}} \cdot\left\{\sum_{k}\left(\frac{\partial c_{i j}}{\partial x_{k}} \cdot a_{k}-\frac{\partial a_{i}}{\partial x_{k}} \cdot c_{k j}-\frac{\partial a_{j}}{\partial x_{k}} \cdot c_{i k}\right)\right\} \cdot
\end{aligned}
$$

Hence $D$ is a derivation of $A$ if and only if

$$
\sum_{k=1}^{n}\left(\frac{\partial c_{i j}}{\partial x_{k}} \cdot a_{k}+\frac{\partial a_{i}}{\partial x_{k}} \cdot c_{j k}+\frac{\partial a_{j}}{\partial x_{k}} \cdot c_{k i}\right)=0
$$

for $1 \leqq i, j \leqq n$. The equations (5) are redundant for $j \geqq i$, so we have proved

Theorem 1. Let $A$ be a nodal noncommutative Jordan algebra in $K$, multiplication being defined by (1). Then a mapping $D$ on $A$ is a derivation of $A$ if and only if $D$ has the form (4) for elements $a_{1}, \cdots, a_{n}$ in $A$ satisfying (5) for $1 \leqq i<j \leqq n$.

Solution of the equations (5) in general seems a formidable task. Even a preliminary simplification of the problem by using (3) to normalize the $c_{i j}$ seems exceedingly complex in the general situation. In the next two sections we treat two special cases: (i) $n$ arbitrary, but all of the $c_{i j}$ in $F 1$; (ii) $n=2$, but $c_{i j}$ arbitrary in $A$.

2. Algebras defined by skew-symmetric bilinear forms. Suppose that all of the $c_{i j}$ are in $F 1$. That is, $c_{i j}=\phi_{i j} 1, \phi_{i j}=-\phi_{j i}$ in $F$, not all $\phi_{i j}$ zero, where $x_{i} x_{j}=x_{i} \cdot x_{j}+\phi_{i j} 1$. There is a unique skew-symmetric bilinear form $\phi$ defined on the $n$-dimensional space $M=\sum_{i=1}^{n} F x_{i}$ (equivalently, defined on $N / N \cdot N$ ) such that $\phi\left(x_{i}, x_{j}\right)=\phi_{i j}$. Then

$$
f g=f \cdot g+\sum_{i, j} \frac{\partial f}{\partial x_{i}} \cdot \frac{\partial g}{\partial x_{j}} \cdot \phi\left(x_{i}, x_{j}\right) 1 .
$$

If $x_{i}(i=1, \cdots, n)$ exist for which $c_{i j}=\phi\left(x_{i}, x_{j}\right) 1$, we shall say that $A$ is defined by the skew-symmetric bilinear form $\phi$. (Since $N(N \cdot N) \subseteq N$ by $[9$, Theorem 5] we know that, if $A$ is defined by different skew-symmetric bilinear forms, the forms are equivalent.) Let $2 r(2 \leqq 2 r \leqq n)$ be the rank of $\phi$. 
A change of basis in $M$ gives $\phi\left(x_{i}, x_{r+i}\right)=1=-\phi\left(x_{r+i}, x_{i}\right)$ for $i=1, \cdots, r$; $\phi\left(x_{i}, x_{j}\right)=0$ otherwise. The form $\phi$ is nondegenerate if and only if $n=2 r$.

We remark that an algebra $A$ defined by a skew-symmetric bilinear form $\phi$ is simple if and only if $\phi$ is nondegenerate. For take the special basis above in $M$. If $n>2 r$, then $c_{i, 2 r+1}=\phi_{i, 2 r+1} 1=0$ for $i=1, \cdots, n$, so that $f x_{2 r+1}=f \cdot x_{2 r+1}$ $=x_{2 r+1} f$ for every $f$ in $A$ by (1). It follows that $C=x_{2 r+1} \cdot A$ is an ideal of $A$, $C \neq 0, C \neq A$. If $n=2 r$, we shall see in $\S 4$ that $A$ is a simple nodal noncommutative Jordan algebra associated in a specific way with one of the algebras in a general class of simple Lie algebras of characteristic $p$. Of course the simplicity of $A$ may also be established directly in this particular case.

If we take the special basis above for $M$ in an algebra $A$ defined by a skew-symmetric form, equations (5) become

$$
\begin{aligned}
\frac{\partial a_{i}}{\partial x_{r+j}} & =\frac{\partial a_{j}}{\partial x_{r+i}}, & i, j & =1, \cdots, r ; \\
\frac{\partial a_{r+i}}{\partial x_{j}} & =\frac{\partial a_{r+j}}{\partial x_{i}}, & i, j & =1, \cdots, r ; \\
\frac{\partial a_{i}}{\partial x_{j}}+\frac{\partial a_{r+j}}{\partial x_{r+i}} & =0, & i, j & =1, \cdots, r ;
\end{aligned}
$$

and

$$
\frac{\partial a_{j}}{\partial x_{i}}=0, \quad 1 \leqq i \leqq 2 r ; 2 r+1 \leqq j \leqq n .
$$

Equations (6), (7), (8) involve only $a_{1}, \cdots, a_{2 r}$. Hence the elements $a_{2 r+k}(k=1, \cdots, n-2 r)$ are arbitrary in $F\left[x_{2 r+1}, \cdots, x_{n}\right]$ by $(9)$.

LeMmA 1. Let $f$ be in $B_{n}=F\left[x_{1}, \cdots, x_{n}\right]$. For any $i(1 \leqq i \leqq n)$, write $R_{i}=F\left[x_{1}, \cdots, x_{i-1}, x_{i+1}, \cdots, x_{n}\right]$ so that $B_{n}=R_{i}\left[x_{i}\right]$. Then there exists $g$ in $B_{n}$ satisfying

$$
\frac{\partial g}{\partial x_{i}}=f
$$

if and only if $f$ is of the form

$$
f=\beta_{0}+\beta_{1} \cdot x_{i}+\beta_{2} \cdot x_{i}^{2}+\cdots+\beta_{p-2} \cdot x_{i}^{p-2},
$$

If $f$ satisfies (11), there is always a solution $g$ of (10) having the form

$$
g=x_{i} \cdot h,
$$$$
h \in B_{n} .
$$

If $g$ is one solution of (10), then $g^{*}$ is a solution if and only if

$$
g^{*}=g+h \text {, }
$$

$h \in R_{i}$ 
The proof is straightforward.

Using the lemma, we give an inductive proof that the solutions of (6)-(9) are

$$
\begin{aligned}
a_{i} & =\frac{\partial g}{\partial x_{r+i}}+\sigma_{i} \cdot x_{r+i}^{p-1} & \text { for } i=1, \cdots, r \\
a_{r+i} & =-\frac{\partial g}{\partial x_{i}}+\sigma_{r+i} \cdot x_{i}^{p-1} & \text { for } i=1, \cdots, r
\end{aligned}
$$

and

$$
a_{2 r+k}=\sigma_{2 r+k} \quad \text { for } k=1, \cdots, n-2 r,
$$

for arbitrary $g$ in $A$ and arbitrary $\sigma_{i}, \sigma_{r+i}, \sigma_{2 r+k}$ in $F\left[x_{2 r+1}, \cdots, x_{n}\right]$. Clearly any elements $a_{1}, \cdots, a_{n}$ given by (14) and (15) satisfy equations (6)-(9). We are concerned only with establishing the fact that, if $a_{1}, \cdots, a_{2 r}$ satisfy (6)-(8), then $a_{1}, \cdots, a_{2 r}$ have the form given in (14).

Let $a_{1}=\beta_{0}+\beta_{1} \cdot x_{r+1}+\cdots+\beta_{p-2} \cdot x_{r+1}^{p-2}+\beta_{p-1} \cdot x_{r+1}^{p-1}, \beta_{j} \in R_{r+1}$, so that, for any $k \neq r+1$,

$$
\frac{\partial a_{1}}{\partial x_{k}}=\frac{\partial \beta_{0}}{\partial x_{k}}+\cdots+\frac{\partial \beta_{p-1}}{\partial x_{k}} \cdot x_{r+1}^{p-1} .
$$

Then, putting $i=1$ in (6) and (8), we have

$$
\frac{\partial \beta_{p-1}}{\partial x_{k}}=0 \quad \text { for } k=1, \cdots, r, r+2, \cdots, 2 r
$$

by (11). Also $\partial \beta_{p-1} / \partial x_{r+1}=0$. By the lemma there exist $g \in B_{n}$ and $\sigma_{1} \in F\left[x_{2 r+1}, \cdots, x_{n}\right]$ such that $a_{1}=\partial g / \partial x_{r+1}+\sigma_{1} \cdot x_{r+1}^{p-1}$. For any $t(2 \leqq t \leqq 2 r)$ we assume that (14) holds for $i=1, \cdots, t-1$. There are two cases to be considered: $t \leqq r$, and $t \geqq r+1$. If $t \leqq r$, we replace $i$ in (6) by $t$, and obtain

$$
\frac{\partial a_{t}}{\partial x_{r+j}}=\frac{\partial^{2} g}{\partial x_{r+t} \partial x_{r+j}} \quad \text { for } j=1, \cdots, t-1,
$$

and

$$
\frac{\partial a_{t}}{\partial x_{r+j}}=\frac{\partial a_{j}}{\partial x_{r+t}} \quad \text { for } j=t+1, \cdots, r .
$$

It follows that $a_{t}=\partial g / \partial x_{r+t}+f$, where

$$
f=\frac{\partial h}{\partial x_{r+t}}+\sigma_{t} \cdot x_{r+t}^{p-1}
$$

for some $h$ satisfying $\partial h / \partial x_{r+j}=0$ for $j=1, \cdots, t-1$, and some $\sigma_{t}$ satisfying 


$$
\frac{\partial \sigma_{t}}{\partial x_{k}}=0
$$

for $k=r+1, \cdots, 2 r$. Write $g^{*}=g+h$. Then $\partial g^{*} / \partial x_{r+i}=\partial g / \partial x_{r+i}$ for $i=1, \cdots, t-1$, so that the inductive hypothesis remains valid with $g^{*}$ replacing $g$. Also $a_{t}=\partial g^{*} / \partial x_{r+t}+\sigma_{t} \cdot x_{r+t}^{p-1}$ for $\sigma_{t}$ satisfying (16) for $k=r+1, \cdots$, $2 r$. Putting $i=t$ in (8), we have

$$
\frac{\partial \sigma_{t}}{\partial x_{j}} \cdot x_{r+t}^{p-1}=-\frac{\partial}{\partial x_{r+t}}\left(a_{r+j}+\frac{\partial g^{*}}{\partial x_{j}}\right),
$$

so (16) holds also for $k=1, \cdots, r$. That is, $\sigma_{t} \in F\left[x_{2 r+1}, \cdots, x_{n}\right]$, as desired. In the second case $(t \geqq r+1)$, we write $t=r+s$. Then the assumption of the induction is that the first line of (14) holds for $i=1, \cdots, r$, and the second line for $i=1, \cdots, s-1$. Putting $j=s$ in (8), we have $a_{r+s}=-\partial g / \partial x_{\imath}+f$, where

$$
\frac{\partial f}{\partial x_{k}}=0
$$

for $k=r+1, \cdots, 2 r$. Also $j=s$ in (7) yields (17) for $k=1, \cdots, s-1$, and

$$
\frac{\partial f}{\partial x_{i}}=\frac{\partial}{\partial x_{i}}\left(a_{r+i}+\frac{\partial g}{\partial x_{i}}\right)
$$

for $i=s+1, \cdots, r$. Hence $f=-\partial h / \partial x_{s}+\sigma_{r+s} \cdot x_{s}^{p-1}$ for some $h$ satisfying $\partial h / \partial x_{k}=0$ for $k=1, \cdots, s-1, r+1, \cdots, 2 r$, and some $\sigma_{r+*}$ satisfying

$$
\frac{\partial \sigma_{r+s}}{\partial x_{k}}=0 \quad \text { for } k=1, \cdots, 2 r .
$$

Write $g^{*}=g+h$. Then $\partial g^{*} / \partial x_{k}=\partial g / \partial x_{k}$ for $k=1, \cdots, s-1, r+1, \cdots, 2 r$, so that the inductive hypothesis remains valid with $g^{*}$ replacing $g$. Also $a_{r+s}=-\partial g^{*} / \partial x_{s}+\sigma_{r+s} \cdot x_{s}^{p-1}$ with $\sigma_{r+s} \in F\left[x_{2 r+1}, \cdots, x_{n}\right]$ by (18). We have established equations (14).

Now any $D$ given by (4), (14), and (15) determines $g$ modulo $F\left[x_{2 r+1}, \cdots, x_{n}\right]$ and the $\sigma_{j}(j=1, \cdots, n)$ uniquely. Hence we have proved

TheOREM 2. Let $A$ be a nodal noncommutative Jordan algebra of dimension $p^{n}$ which is defined by a skew-symmetric bilinear form $\phi$ of rank $2 r$. Then the derivation algebra $D(A)$ of $A$ has dimension

$$
p^{n}-p^{n-2 r}+n p^{n-2 r}=p^{n-2 r}\left(p^{2 r}-1+n\right) .
$$

The $x_{i}$ in $A$ may be chosen so that the derivations $D$ of $A$ have the form (4) with $a_{k}$ as in (14) and (15).

Let $D$ be given by (4) and $E$ by 


$$
f E=\sum_{k=1}^{n} \frac{\partial f}{\partial x_{k}} \cdot b_{k}
$$

for $b_{k}$ in $A$. Then it is well-known $\left[4\right.$, p. 107] that $[D, E]=C=\left(c_{1}, \cdots, c_{n}\right)$ where

$$
c_{i}=\sum_{j=1}^{n}\left(\frac{\partial a_{i}}{\partial x_{j}} \cdot b_{j}-\frac{\partial b_{i}}{\partial x_{j}} \cdot a_{j}\right), \quad i=1, \cdots, n .
$$

It is easily seen that, in case $n>2 r$ above, there are many ideals in $D(A)$. If $n=2 r$, however, (that is, if $A$ is defined by a nondegenerate $\phi$ ) we have (14) with $\sigma_{k} \in F 1(=F)$ for $k=1, \cdots, 2 r$. Writing $D\left(g ; \sigma_{1}, \cdots, \sigma_{2 r}\right)$ for $D$ in (14), we obtain

$$
\left[D\left(f ; \rho_{1}, \cdots, \rho_{2 r}\right), D\left(g ; \sigma_{1}, \cdots, \sigma_{2 r}\right)\right]=D(h ; 0, \cdots, 0)
$$

from (20), where

$$
\begin{aligned}
h= & \sum_{j=1}^{r}\left\{\left(\rho_{j} \sigma_{r+j}-\rho_{r+j} \sigma_{j}\right) x_{j}^{p-1} \cdot x_{r+j}^{p-1}+\left(\frac{\partial f}{\partial x_{j}} \cdot \frac{\partial g}{\partial x_{r+j}}-\frac{\partial f}{\partial x_{r+j}} \cdot \frac{\partial g}{\partial x_{j}}\right)\right. \\
& \left.+\left(\sigma_{r+j} \frac{\partial f}{\partial x_{r+j}}-\rho_{r+j} \frac{\partial g}{\partial x_{r+j}}\right) \cdot x_{j}^{p-1}+\left(\sigma_{j} \frac{\partial f}{\partial x_{j}}-\rho_{j} \frac{\partial g}{\partial x_{j}}\right) \cdot x_{r+j}^{p-1}\right\} .
\end{aligned}
$$

We recognize $D(g)=D(g ; 0, \cdots, 0)$ as any element of the algebra $V_{0 r}$ of Albert and Frank [1, p. 127]. Let $\tilde{A}$ be the subspace of $B_{n}$ consisting of all elements of $B_{n}$ for which the coefficient of $x^{p-1} \cdot x_{2}^{p-1} \cdots \cdots x_{n}^{p-1}$ is zero. The $\left(p^{2 r}-2\right)$-dimensional simple Lie algebra $V_{r}$ of Albert and Frank consists of all $D(g)$ with $g \in \widetilde{A}$. It is known [1, pp. 127, 128] that $V_{r}$ is the derived algebra $V_{0 r}^{\prime}$ of $V_{0 r}$ and that $\partial f / \partial x_{j} \cdot \partial g / \partial x_{r+j}-\partial f / \partial x_{r+j} \cdot \partial g / \partial x_{j}$ is in $\tilde{A}$. It follows from (11) that $\partial f / \partial x_{r+j} \cdot x_{j}^{p-1} \in \tilde{A}$, etc. Hence only the first term within the braces in (22) could fail to be in $\tilde{A}$. If $r>1, h$ in (22) is in $\tilde{A}$, and $D(h) \in V_{r}$. Hence $V_{r}=V_{0 r}^{\prime} \subseteq D(A)^{\prime} \subseteq V_{r}$ if $r>1$. If $r=1$, there exists $h$ in (22) which is not in $\tilde{A}$, so that $D(A)^{\prime} \subseteq V_{01}$ but $D(A)^{\prime} \neq V_{1}$. However, $V_{1}=V_{1}^{\prime} \subseteq D(A)^{\prime \prime}$ $\subseteq V_{01}^{\prime}=V_{1}$. Of course $D(A)^{\prime \prime}=V_{r}$ for $r>1$ also.

Thus we obtain the following intrinsic characterization of the $\left(p^{2 r}-2\right)$ dimensional simple Lie algebras $V_{r}$ of Albert and Frank:

THeOREM 3. A Lie algebra $L$ is a simple algebra $V_{r}$ if and only if there is a $p^{2 r}$-dimensional (simple) nodal noncommutative Jordan algebra $A$ defined by a nondegenerate skew-symmetric bilinear form such that $L \cong D(A)^{\prime}$ in case $r>1$, and $L \cong D(A)^{\prime \prime}$ in case $r=1$ (actually $L \cong D(A)^{\prime \prime}$ in both cases).

A second characterization of the algebras $V_{r}$ results from the following observation concerning Lie-admissible algebras.

In any nonassociative algebra $A, x y=x \cdot y+[x, y] / 2$. Therefore a linear transformation $D$ on $A$ is a derivation if and only if $D$ is a derivation of both $A^{+}$and $A^{-}$, where $A^{-}$is the anticommutative algebra attached to $A$ having the product $[x, y]$. That is, 


$$
D(A)=D\left(A^{+}\right) \cap D\left(A^{-}\right) .
$$

Now in any nodal noncommutative Jordan algebra $A$ in $K$

$$
[f, g]=\sum_{i, j} \frac{\partial f}{\partial x_{i}} \cdot \frac{\partial g}{\partial x_{j}} \cdot 2 c_{i j},
$$

and $D$ in (4) is a derivation of $A$ if and only if $D$ is a derivation of $A^{-}$.

If $A^{-}$is a Lie algebra, then the mappings ad $g / 2$ defined by

$$
f \rightarrow \frac{1}{2}[f, g]=\sum_{i} \frac{\partial f}{\partial x_{i}} \cdot\left(\sum_{j} \frac{\partial g}{\partial x_{j}} \cdot c_{i j}\right)
$$

are inner derivations of $A^{-}$, and therefore derivations of $A$ since they are of the form (4) with

$$
a_{i}=\sum_{j=1}^{n} \frac{\partial g}{\partial x_{j}} \cdot c_{i j}, \quad i=1, \cdots, n .
$$

The set ad $A$ of all inner derivations (25) of $A^{-}$, being an ideal of $D\left(A^{-}\right)$, is an ideal of $D(A)$ by (23).

We use the Jacobi identity

$$
\begin{aligned}
0= & {[[f, g], h]+[[g, h], f]+[[h, f], g] } \\
= & 2 \sum_{i, j}\left\{\left[\frac{\partial f}{\partial x_{i}} \cdot \frac{\partial g}{\partial x_{j}} \cdot c_{i j}, h\right]+\left[\frac{\partial g}{\partial x_{i}} \cdot \frac{\partial h}{\partial x_{j}} \cdot c_{i j}, f\right]+\left[\frac{\partial h}{\partial x_{i}} \cdot \frac{\partial f}{\partial x_{j}} \cdot c_{i j}, g\right]\right\} \\
= & 4 \sum_{i, j, k, t}\left\{\frac{\partial}{\partial x_{t}}\left(\frac{\partial f}{\partial x_{i}} \cdot \frac{\partial g}{\partial x_{j}} \cdot c_{i j}\right) \cdot \frac{\partial h}{\partial x_{k}} \cdot c_{t k}\right. \\
& \left.+\frac{\partial}{\partial x_{t}}\left(\frac{\partial g}{\partial x_{i}} \cdot \frac{\partial h}{\partial x_{j}} \cdot c_{i j}\right) \cdot \frac{\partial f}{\partial x_{k}} \cdot c_{t k}+\frac{\partial}{\partial x_{t}}\left(\frac{\partial h}{\partial x_{i}} \cdot \frac{\partial f}{\partial x_{j}} \cdot c_{i j}\right) \cdot \frac{\partial g}{\partial x_{k}} \cdot c_{t k}\right\} \\
= & 4 \sum_{i, j, k, t}\left(\frac{\partial^{2} f}{\partial x_{t} \partial x_{i}} \cdot \frac{\partial g}{\partial x_{j}} \cdot \frac{\partial h}{\partial x_{k}} \cdot c_{i j} \cdot c_{t k}+\frac{\partial f}{\partial x_{i}} \cdot \frac{\partial^{2} g}{\partial x_{t} \partial x_{j}} \cdot \frac{\partial h}{\partial x_{k}} \cdot c_{i j} \cdot c_{t k}\right. \\
& +\frac{\partial f}{\partial x_{i}} \cdot \frac{\partial g}{\partial x_{j}} \cdot \frac{\partial h}{\partial x_{k}} \cdot \frac{\partial c_{i j}}{\partial x_{t}} \cdot c_{t k}+\frac{\partial^{2} g}{\partial x_{j} \partial x_{i}} \cdot \frac{\partial h}{\partial x_{k}} \cdot \frac{\partial f}{\partial x_{i}} \cdot c_{t k} \cdot c_{j i}+\frac{\partial g}{\partial x_{k}} \cdot \frac{\partial^{2} h}{\partial x_{i} \partial x_{t}} \cdot \frac{\partial f}{\partial x_{j}} \cdot c_{k t} \cdot c_{i j} \\
& +\frac{\partial g}{\partial x_{j}} \cdot \frac{\partial h}{\partial x_{k}} \cdot \frac{\partial f}{\partial x_{i}} \cdot \frac{\partial c_{j k}}{\partial x_{t}} \cdot c_{t i}+\frac{\partial^{2} h}{\partial x_{t} \partial x_{i}} \cdot \frac{\partial f}{\partial x_{j}} \cdot \frac{\partial g}{\partial x_{k}} \cdot c_{i j} \cdot c_{t k}+\frac{\partial h}{\partial x_{k}} \cdot \frac{\partial^{2} f}{\partial x_{i} \partial x_{t}} \cdot \frac{\partial g}{\partial x_{j}} \cdot c_{k t} \cdot c_{i j} \\
& \left.+\frac{\partial h}{\partial x_{k}} \cdot \frac{\partial f}{\partial x_{i}} \cdot \frac{\partial g}{\partial x_{j}} \cdot \frac{\partial c_{k i}}{\partial x_{t}} \cdot c_{t j}\right) \\
= & 4 \sum_{i, j, k} \frac{\partial f}{\partial x_{i}} \cdot \frac{\partial g}{\partial x_{j}} \cdot \frac{\partial h}{\partial x_{k}} \cdot \sum_{t}\left(\frac{\partial c_{i j}}{\partial x_{i}} \cdot c_{t k}+\frac{\partial c_{j k}}{\partial x_{t}} \cdot c_{t i}+\frac{\partial c_{k i}}{\partial x_{i}} \cdot c_{t j}\right)
\end{aligned}
$$


to see that $A^{-}$is a Lie algebra if and only if

$$
\sum_{t=1}^{n}\left(\frac{\partial c_{i j}}{\partial x_{t}} \cdot c_{t k}+\frac{\partial c_{j k}}{\partial x_{t}} \cdot c_{t i}+\frac{\partial c_{k i}}{\partial x_{t}} \cdot c_{t j}\right)=0
$$

for $i, j, k=1, \cdots, n$. The equations (27) are redundant for $i \geqq j$ and for $j \geqq k$. Hence $A^{-}$is a Lie algebra if and only if (27) holds for $1 \leqq i<j<k \leqq n$. (It follows that $A^{-}$is a Lie algebra in case $n=2$.)

The equations (27) are obviously satisfied in any algebra $A$ defined by a skew-symmetric bilinear form $\phi$. Using the basis employed before in $M$ $=\sum_{i} F x_{i}$, we have ad $g / 2$ in the form (4) with $a_{k}$ given by (14) and (15) with $\sigma_{1}=\cdots=\sigma_{n}=0$. Therefore ad $A$ is an ideal of dimension $p^{n-2 r}\left(p^{2 r}-1\right)$ in $D(A)$ which is of dimension $p^{n-2 r}\left(p^{2 r}-1+n\right)$. If $\phi$ is nondegenerate, so that $n=2 r$, then ad $A$ is the (nonsimple) algebra $V_{\text {or }}$ of all $D(g)$ for $g \in A$.

Theorem 4. A Lie algebra $L$ is a simple Lie algebra $V_{r}$ if and only if there is a $p^{2 r}$-dimensional (simple) nodal noncommutative Jordan algebra $A$ defined by a nondegenerate skew-symmetric bilinear form such that $L \cong(\operatorname{ad} A)^{\prime}$.

3. The case $n=2$. Let $A$ in $K$ have least possible dimension $p^{2}$. Then it is known $[5, \S 3]$ that $A$ is simple. The vector space of $A$ coincides with $B_{2}=F\left[x_{1}, x_{2}\right], x_{1}^{p}=x_{2}^{p}=0$, and multiplication in $A$ is defined by

$$
f g=f \cdot g+\left(\frac{\partial f}{\partial x_{1}} \cdot \frac{\partial g}{\partial x_{2}}-\frac{\partial f}{\partial x_{2}} \cdot \frac{\partial g}{\partial x_{1}}\right) \cdot c
$$

where $c$ has an inverse $c^{-1}$ in $B_{2}$. Also (5) reduces to the single equation

$$
\frac{\partial c}{\partial x_{1}} \cdot a_{1}-\frac{\partial a_{1}}{\partial x_{1}} \cdot c+\frac{\partial c}{\partial x_{2}} \cdot a_{2}-\frac{\partial a_{2}}{\partial x_{2}} \cdot c=0,
$$

which is equivalent to

$$
\frac{\partial}{\partial x_{1}}\left(c^{-1} \cdot a_{1}\right)+\frac{\partial}{\partial x_{2}}\left(c^{-1} \cdot a_{2}\right)=0 .
$$

If, given $D$ in (4), we write $b \cdot D$ for the derivation

$$
f \rightarrow \sum_{k=1}^{n} \frac{\partial f}{\partial x_{k}} \cdot\left(b \cdot a_{k}\right)
$$

of $B_{n}$ (that is, $\left.b \cdot D=\left(b \cdot a_{1}, \cdots, b \cdot a_{n}\right)\right)$, and if we write $\delta(D)$ for the divergence [3, p. 715]

$$
\delta(D)=\sum_{k=1}^{n} \frac{\partial a_{k}}{\partial x_{k}}
$$

then condition (29) becomes 


$$
\delta\left(c^{-1} \cdot D\right)=0 .
$$

Hence $D$ is a derivation of $A$ if and only if $c^{-1} \cdot D$ is in the $\left(p^{2}+1\right)$-dimensional Lie algebra $M_{2}$ of derivations of $B_{2}$ having divergence zero [3]. But $D \leftrightarrow c^{-1} \cdot D$ is a vector space isomorphism, so the derivation algebra $D(A)$ is a $\left(p^{2}+1\right)$ dimensional algebra $D(A)=c \cdot M_{2}$. If $c=1$, so that $A$ is defined by a (nondegenerate) form $\phi$, then $D(A)=M_{2}$ consists of the derivations (4) of $B_{2}$ given by (14) with $r=1$, while $D(A)^{\prime}=V_{01}$, and $D(A)^{\prime \prime}=V_{1}$ is simple. More generally two distinct situations arise, depending upon whether $c^{-1}$ is in $\tilde{A}$ or not.

We digress momentarily to point out that (30) defines Lie algebras which generalize the algebras $M_{n}$ of Frank [3], not only for $n=2$, but for general $n$. Let $c$ be any invertible element of $B_{n}$, and let $D$ range over the derivations of $B_{n}$ satisfying (30). The set $c \cdot M_{n}$ of these derivations of $B_{n}$ is a Lie algebra of dimension $(n-1) p^{n}+1$ since $g$ in $B_{n}$ implies

$$
\delta(g \cdot[D, E])=\delta(\delta(g \cdot D) \cdot E)-\delta(\delta(g \cdot E) \cdot D),
$$

generalizing $[3$, Lemma 2]. For (20) gives

$$
\begin{aligned}
\delta(\delta(g \cdot D) \cdot E)-\delta(\delta(g \cdot E) \cdot D) \\
=\sum_{j} \frac{\partial}{\partial x_{j}}\left\{\sum_{i}\left(\frac{\partial\left(g \cdot a_{i}\right)}{\partial x_{i}} \cdot b_{j}-\frac{\partial\left(g \cdot b_{i}\right)}{\partial x_{i}} \cdot a_{j}\right)\right\} \\
=\sum_{i, j}\left(\frac{\partial g}{\partial x_{i}} \cdot \frac{\partial a_{i}}{\partial x_{j}} \cdot b_{j}+g \cdot \frac{\partial^{2} a_{i}}{\partial x_{j} \partial x_{i}} \cdot b_{j}-\frac{\partial g}{\partial x_{i}} \cdot \frac{\partial b_{i}}{\partial x_{j}} \cdot a_{j}-g \cdot \frac{\partial^{2} b_{i}}{\partial x_{j} \partial x_{i}} \cdot a_{j}\right) \\
=\sum_{i} \frac{\partial g}{\partial x_{i}} \cdot c_{i}+g \cdot \sum_{i} \frac{\partial c_{i}}{\partial x_{i}} \\
=\delta(g \cdot[D, E]) .
\end{aligned}
$$

Putting $g=c^{-1}$ in (31), we see that $[D, E]$ satisfies (30) in case $D$ and $E$ do, or $c \cdot M_{n}$ is a Lie algebra. Also

$$
\left(c \cdot M_{n}\right)^{\prime} \subseteq c \cdot M_{n}^{\prime} .
$$

For suppose that $D$ and $E$ satisfy (30). Writing $g=c^{-1}$, we have

$$
\begin{aligned}
\sum_{j=1}^{n} & \frac{\partial}{\partial x_{j}}\left(g \cdot a_{i} \cdot b_{j}-g \cdot b_{i} \cdot a_{j}\right) \\
& =g \cdot \sum_{j=1}^{n}\left(\frac{\partial a_{i}}{\partial x_{j}} \cdot b_{j}-\frac{\partial b_{i}}{\partial x_{j}} \cdot a_{j}\right)+a_{i} \cdot \delta(g \cdot E)-b_{i} \cdot \delta(g \cdot D) \\
& =g \cdot c_{i}
\end{aligned}
$$

for $c_{i}$ in (20). Hence $g \cdot[D, E]=\left(h_{1}, \cdots, h_{n}\right)$ where 


$$
h_{i}=\sum_{j \neq i} \frac{\partial}{\partial x_{j}}\left(g \cdot a_{i} \cdot b_{j}-g \cdot b_{i} \cdot a_{j}\right) .
$$

Hence $g \cdot[D, E]$ is in the algebra $T_{n}=S_{n}$ defined by Frank [3, Lemma 3]. But $S_{n}=M_{n}^{\prime}$ (because $S_{n}$ is simple in case $n>2$ and by our earlier remarks in case $n=2)$. Hence $[D, E] \in c \cdot M_{n}^{\prime}$, establishing (32). It follows that $c \cdot M_{n}^{\prime}$ $=c \cdot S_{n}$ is a Lie algebra (of dimension $(n-1)\left(p^{n}-1\right)$, the known dimension of $\left.S_{n}\right)$, for $\left(c \cdot M_{n}^{\prime}\right)^{\prime} \subseteq\left(c \cdot M_{n}\right)^{\prime} \subseteq c \cdot M_{n}^{\prime}$ by $(32)$.

Returning to the case $n=2$, we shall prove that $D(A)^{\prime \prime}=(\operatorname{ad} A)^{\prime} \cong V_{1}$ is a simple Lie algebra of dimension $p^{2}-2$ if $c^{-1} \in \tilde{A}$, whereas $D(A)^{\prime}=\operatorname{ad} A$ is a simple Lie algebra of dimension $p^{2}-1$ if $c^{-1} \notin \tilde{A}$. We begin with a normalization of $c$ by proper choice of $x_{i}$ in $A$.

THeOREm 5. Let $A$ be a (simple) nodal noncommutative Jordan algebra of dimension $p^{2}$ in $K$ so that multiplication in $A$ is defined by (28). Then $x_{i}$ may be chosen in $A$ so that $c$ is in the form

$$
c=1+\alpha x_{1}^{p-1} \cdot x_{2}^{p-1},
$$

$\alpha \in F$.

According as $c^{-1}$ is or is not in $\tilde{A}$ (for any choice of $x_{i}$ ), we have $\alpha=0$ or $\alpha \neq 0$ in (33).

Proof. Write $c^{-1}=\beta_{0}+\beta_{1} \cdot x_{2}+\cdots+\beta_{p-2} \cdot x_{2}^{p-2}+\beta_{p-1} \cdot x_{2}^{p-1}, \quad \beta_{j} \in F\left[x_{1}\right]$. Then $\beta_{0}^{-1}$ exists, and $c^{-1} \cdot\left(1-\beta_{0}^{-1} \cdot \beta_{p-1} \cdot x_{2}^{p-1}\right)=\beta_{0}+\beta_{1} \cdot x_{2}+\cdots+\beta_{p-2} \cdot x_{2}^{p-2}$. But then (11) and (12) imply that there exists $y_{2}=x_{2} \cdot h$ such that

$$
\frac{\partial y_{2}}{\partial x_{2}}=c^{-1} \cdot\left(1-\beta_{0}^{-1} \cdot \beta_{p-1} \cdot x_{2}^{p-1}\right) \text {. }
$$

Now $\partial y_{2} / \partial x_{2}=x_{2} \cdot \partial h / \partial x_{2}+h$ implies that $h$ and $\beta_{0}$ are congruent modulo $N$. Hence $h^{-1}$ exists, so that $y_{2}=\delta x_{2}+n, n \in N \cdot N, \delta \neq 0$. Let $y_{1}=x_{1}$. Then $A=F\left[y_{1}, y_{2}\right], y_{1}^{p}=y_{2}^{p}=0$. Now $y_{2}^{p-1}=x_{2}^{p-1} \cdot h^{p-1}$, so that $x_{2}^{p-1} \in A \cdot y_{2}^{p-1}$. But every element of $A \cdot y_{2}^{p-1}$ has the form $\rho \cdot y_{2}^{p-1}$ for $\rho \in F\left[y_{1}\right]$. Then (3) and (34) imply $\left[y_{1}, y_{2}\right] / 2=\left(\partial y_{2} / \partial x_{2}\right) \cdot c=1-\beta_{0}^{-1} \cdot \beta_{p-1} \cdot x_{2}^{p-1}=1+\sigma \cdot y_{2}^{p-1}$ where

$$
\sigma=-\beta_{0}^{-1} \cdot \beta_{p-1} \cdot \rho \in F\left[y_{1}\right] .
$$

That is, we may as well take $c$ in (28) in the form

$$
c=1+\sigma \cdot x_{2}^{p-1}, \quad \sigma \in F\left[x_{1}\right]
$$

Now $\sigma=\alpha_{0} 1+\alpha_{1} x_{1}+\cdots+\alpha_{p-2} x_{1}^{p-2}+\alpha_{p-1} x_{1}^{p-1}$ for $\alpha_{j} \in F$. Then

$$
\begin{aligned}
c^{-1} \cdot\left(1+\alpha_{p-1} x_{1}^{p-1} \cdot x_{2}^{p-1}\right) & =\left(1-\sigma \cdot x_{2}^{p-1}\right) \cdot\left(1+\alpha_{p-1} x_{1}^{p-1} \cdot x_{2}^{p-1}\right) \\
& =1-\alpha_{0} x_{2}^{p-1}-\alpha_{1} x_{1} \cdot x_{2}^{p-1}-\cdots-\alpha_{p-2} x_{1}^{p-2} \cdot x_{2}^{p-1} .
\end{aligned}
$$

By (11) and (12) there exists 


$$
y_{1}=x_{1} \cdot\left(1+\pi \cdot x_{2}^{p-1}\right),
$$

such that

$$
\frac{\partial y_{1}}{\partial x_{1}}=c^{-1} \cdot\left(1+\alpha x_{1}^{p-1} \cdot x_{2}^{p-1}\right)
$$

where we have written $\alpha$ for $\alpha_{p-1} \in F$. Then $y_{1}^{p-1}=x_{1}^{p-1} \cdot\left(1-\pi \cdot x_{2}^{p-1}\right)$, or $x_{1}^{p-1}=y_{1}^{p-1} \cdot\left(1+\pi \cdot x_{2}^{p-1}\right)$. Let $y_{2}=x_{2}$. Then $A=F\left[y_{1}, y_{2}\right]$, while (3) and (35) imply that $\left[y_{1}, y_{2}\right] / 2=\left(\partial y_{1} / \partial x_{1}\right) \cdot c=1+\alpha x_{1}^{p-1} \cdot x_{2}^{p-1}=1+\alpha y_{1}^{p-1} \cdot\left(1+\pi \cdot y_{2}^{p-1}\right) \cdot y_{2}^{p-1}$ $=1+\alpha y_{1}^{p-1} \cdot y_{2}^{p-1}$. That is, we may take $c$ in the form (33).

The final statement in the theorem could probably be established by a careful analysis of the argument above. Instead we note that, for any choice of $x_{i}, V_{01}$ consists of all $D(g)$ with $g \in A$, and $V_{1}=V_{01}^{\prime}$ of all $D(g)$ for which $g \in \tilde{A}$, while $[D(f), D(g)]=D(h)$ where

$$
h=\frac{\partial f}{\partial x_{1}} \cdot \frac{\partial g}{\partial x_{2}}-\frac{\partial f}{\partial x_{2}} \cdot \frac{\partial g}{\partial x_{1}} .
$$

Since 1 is in $\tilde{A}$, as well as being of the form (36), it follows that $\tilde{A}$ consists of all linear combinations of elements of the form (36). By (24) we have $[f, g] / 2$ $=h \cdot c$ for $h$ in (36), or $[A, A]=\tilde{A} \cdot c$. Hence $c^{-1} \in \tilde{A}$ if and only if $1 \in[A, A]$. But the latter condition is independent of the choice of $x_{i}$. Hence, for any choice of $x_{i}, c^{-1} \in \tilde{A}$ if and only if the $c^{-1}=1-\alpha x_{1}^{p-1} \cdot x_{2}^{p-1}$ given by (33) is in $\widetilde{A}$.

One point in the proof of the next theorem is deferred to the final section where we consider the simple Lie algebras $L(G, \delta, f)$.

THEOREM 6. Let $A$ be a (simple) nodal noncommutative Jordan algebra of dimension $p^{2}$ in $K$ so that multiplication in $A$ is defined by (28). Then

$$
D(A)=c \cdot M_{2}
$$

where $M_{2}$ is the $\left(p^{2}+1\right)$-dimensional algebra consisting of all derivations (4) of $B_{2}$ given by (14) with $r=1$, and $D(A)^{\prime \prime}$ is simple. If $c^{-1} \in \tilde{A}$, then $D(A) \cong M_{2}$, $D(A)^{\prime} \cong V_{01}$, and $D(A)^{\prime \prime} \cong V_{1}$ is of dimension $p^{2}-2$. If $c^{-1} \notin \widetilde{A}$, then $D(A)^{\prime}$ $=D(A)^{\prime \prime}=c \cdot M_{2}^{\prime}$ is of dimension $p^{2}-1$.

Proof. The case $c^{-1} \in \tilde{A}$ has already been established since we may take $c=1$ by Theorem 5. Suppose that $c^{-1} \notin \tilde{A}$ so we may take $c$ in the form (33) with $\alpha \neq 0$. We have seen that (37) holds. Hence (32) implies that

$$
\operatorname{dim} D(A)^{\prime} \leqq \operatorname{dim}\left(c \cdot M_{2}^{\prime}\right)=p^{2}-1 .
$$

We shall see in the next section that a class of central simple Lie algebras $L_{0}$ of dimension $p^{2}-1$ is obtained as follows: $L_{0} \cong$ ad $A$ where $A$ has multiplication defined by (28) with $c=\gamma\left(1+x_{1}\right) \cdot\left(1+x_{2}\right)$ for $\gamma \neq 0 \in F$. We trace through 
the steps of the proof of Theorem 5 to see that $x_{i}$ may be chosen in this $A$ so that

$$
\alpha=-\gamma^{p-1}
$$

in (33): $c^{-1}=\gamma^{-1}\left(1+x_{1}\right)^{-1} \cdot\left(1+x_{2}\right)^{-1}=\gamma^{-1}\left(1+x_{1}\right)^{-1}\left(1-x_{2}+x_{2}^{2}-\cdots+x_{2}^{p-1}\right)$ so that $\beta_{0}=\beta_{p-1}=\gamma^{-1}\left(1+x_{1}\right)^{-1}$. Then $y_{1}=x_{1}$,

$$
\begin{aligned}
y_{2} & =\gamma^{-1}\left(1+x_{1}\right)^{-1} \cdot x_{2} \cdot\left(1-\frac{1}{2} x_{2}+\cdots-\frac{1}{p-1} x_{2}^{p-2}\right) \\
y_{2}^{p-1} & =\gamma^{-(p-1)}\left(1+x_{1}\right)^{-(p-1)} \cdot x_{2}^{p-1} \cdot\left(1-\frac{1}{2} x_{2}+\cdots-\frac{1}{p-1} x_{2}^{p-2}\right)^{p-1} \\
& =\gamma^{-(p-1)}\left(1+x_{1}\right)^{-(p-1)} \cdot x_{2}^{p-1},
\end{aligned}
$$

so that $x_{2}^{p-1}=\gamma^{p-1}\left(1+x_{1}\right)^{p-1} \cdot y_{2}^{p-1}=\gamma^{p-1}\left(1+y_{1}\right)^{p-1} \cdot y_{2}^{p-1}$. Then $\alpha$ in (33) is the coefficient of $y_{1}^{p-1} \cdot y_{2}^{p-1}$ in $-\beta_{0}^{-1} \cdot \beta_{p-1} \cdot x_{2}^{p-1}=-x_{2}^{p-1}$; that is, we have (39). Let $H=F(\gamma)$ where $\gamma$ satisfies (39). Then $(\operatorname{ad} A)_{H}=\operatorname{ad}\left(A_{H}\right) \cong L_{0}$ (an algebra defined over $H$ ) is simple and of dimension $p^{2}-1$ over $H$. Hence ad $A$ is simple and of dimension $p^{2}-1$ over $F$. But ad $A \subseteq D(A)$ implies $p^{2}-1$ $=\operatorname{dim}(\operatorname{ad} A)=\operatorname{dim}(\operatorname{ad} A)^{\prime} \leqq \operatorname{dim} D(A)^{\prime} \leqq p^{2}-1$ by (38). Hence ad $A$ $=D(A)^{\prime}=D(A)^{\prime \prime}=\left(c \cdot M_{2}\right)^{\prime}=c \cdot M_{2}^{\prime}$.

We remark that equality holds in (32) for $n=2$.

4. The simple algebras $L(G, \delta, f)$. The simple Lie algebras $L_{0}$ and $L_{\delta}$ of Albert and Frank [1] have been generalized by Block $\left({ }^{3}\right)$ in [2] to an extensive class of simple Lie algebras $L(G, \delta, f)$. Block has shown [2, Lemma 3] that each $V_{r}$ is an algebra $L(G, \delta, f)$. In this section we prove

THEOREM 7. For any simple Lie algebra $L(G, \delta, f)$ (of characteristic $\neq 2$ ) there exists a simple nodal noncommutative Jordan algebra $A$ in $K$ such that $A^{-}$is a Lie algebra and $L(G, \delta, f) \cong(\operatorname{ad} A)^{\prime}$, an ideal in $D(A)$. Actually $L_{0}$ $\cong$ ad $A$.

If $L(G, \delta, f)$ is simple, then $G=G_{0}+G_{1}+\cdots+G_{m}$ is an elementary $p$ group [2, Theorem 2], so that each $G_{k}$ may be regarded as an $n_{k}$-dimensional vector space over the prime field $F_{p}$ of characteristic $p$. The order of $G$ is $p^{n}$

( ${ }^{3}$ I am indebted to Dr. Block for furnishing me with a copy of his excellent dissertation [2] before its publication. My Theorem 7 was suggested by his Lemma 3 . The following remarks about [2] may be of interest: (i) each of the algebras $V_{m, \mu}$ is isomorphic to $V_{m}$, for $y_{i}=\mu_{i}^{-1} x_{i}, y_{m+i}=\mu_{i}^{-1} x_{m+i}(i=1, \cdots, m)$ implies $\mu_{i} \partial \phi / \partial x_{m+i}=\partial \phi / \partial y_{m+i},-\mu_{i} \partial \phi / \partial x_{i}=-\partial \phi / \partial y_{i}$, and the coefficient of $\left(x_{1} \cdots x_{2 m}\right)^{p-1}$ is zero if and only if the coefficient of $\left(y_{1} \cdots y_{2 m}\right)^{p-1}$ is; (ii) if $F=F_{p}$, the prime field of characteristic $p$, then any simple $L(G, \delta, f)$ for which $G_{0}=0$ is isomorphic to $V_{m}$, for [2, Theorem 4] implies that in each $G_{i}\left\{h(\delta), h\left(\beta_{1}\right), \cdots, h\left(\beta_{k}\right)\right\}$ and $\left\{g\left(\beta_{k+1}, \cdots, g\left(\beta_{r}\right)\right\}\right.$ are linearly independent sets (of elements in $\left.F\right)$ over $F_{p}$, so that $k=0$ and $r=k+1=1$, requiring that each $G_{i}$ be 2-dimensional over $F_{p}$ and that $L(G, \delta, f) \cong V_{m}$ by $[2$, Lemma 3] and (i) above. 
where $n=n_{0}+n_{1}+\cdots+n_{m}$, and we write $q_{-1}=0, q_{k}=n_{0}+n_{1}+\cdots+n_{k}$ $(k=0,1, \cdots, m)$. Let $\sigma_{1}, \cdots, \sigma_{n_{0}}$ be any basis for $G_{0}$ over $F_{p}$. Since $\delta=\delta_{0}$ $+\delta_{1}+\cdots+\delta_{m}$ where $\delta_{0}=0$ and $\delta_{k} \neq 0$ in $G_{k}$ for $k=1, \cdots, m$, we may take a basis $\sigma_{q_{k-1}+1}, \cdots, \sigma_{q_{k-1}}, \delta_{k}$ for $G_{k}$ over $F_{p}(k=1, \cdots, m)$. But then, defining $\sigma_{q_{k}}$ by

$$
\delta_{k}=\sigma_{q_{k-1}+1}+\cdots+\sigma_{q_{k-1}}+\sigma_{q_{k}},
$$

we also have $\sigma_{q_{k-1}+1}, \cdots, \sigma_{q k}$ a basis for $G_{k}$ over $F_{p}(k=1, \cdots, m)$. Then $\sigma_{1}, \cdots, \sigma_{n}$ is a basis for $G$ over $F_{p}$, and any $\alpha \in G$ may be written uniquely in the form

$$
\alpha=\sum_{i=1}^{n} s_{i} \sigma_{i}
$$

Now $L(G, \delta, f)$ is the derived algebra of a Lie algebra $L / F u_{0}$ of dimension $p^{n}-1$ over $F$, where $L$ has a basis consisting of $p^{n}$ elements $u_{\alpha}$ in (1-1) correspondence with the elements $\alpha$ of $G$. By (41) the $u_{\alpha}$ are in (1-1) correspondence with the $n$-tuples $\left(s_{1}, \cdots, s_{n}\right), s_{i} \in F_{p}$, and we shall represent the $u_{\alpha}$ in this way. The skew-symmetric biadditive function $f(\alpha, \beta)$ on $G$ to $F$ may be taken so that $f\left(\alpha_{k}, \beta_{l}\right)=0$ for $k \neq l, \alpha_{k} \in G_{k}, \beta_{l} \in G_{l}(k, l=0,1, \cdots, m)$. Writing $f\left(\sigma_{i}, \sigma_{j}\right)=\alpha_{i j} \in F$, we see that

$$
\alpha_{i j}=0 \text { unless } q_{k-1}+1 \leqq i, j \leqq q_{k} \text { for some } k \quad(0 \leqq k \leqq m) .
$$

Now (41) and $\beta=\sum_{j=1}^{n} t_{j} \sigma_{j}$ imply $f\left(\alpha_{k}, \beta_{k}\right)=\sum_{i, j=q_{k-1}+1}^{q_{k}} s_{i} t_{j} \alpha_{i j}$. Since $\delta=\sum_{k=n_{0}+1}^{n} \sigma_{k}$ by $(40)$, we see that $[2,(4)]$ defines multiplication in $L$ by

$$
\begin{array}{r}
\left(s_{1}, \cdots, s_{n}\right)\left(t_{1}, \cdots, t_{n}\right)=\sum_{i, j=1}^{n_{0}} s_{i} t_{j} \alpha_{i j}\left(s_{1}+t_{1}, \cdots, s_{n}+t_{n}\right) \\
+\sum_{k=1}\left(\sum _ { i , j = q _ { k - 1 } + 1 } ^ { q _ { k } } s _ { i } t _ { j } \alpha _ { i j } \left(s_{1}+t_{1}, \cdots, s_{q_{k-1}}+t_{q_{k-1}}, s_{q_{k-1}+1}+t_{q_{k-1}+1}-1\right.\right. \\
\left.\left.\cdots, s_{q_{k}}+t_{q_{k}}-1, s_{q_{k+1}}+t_{q_{k+1}+1}, \cdots, s_{n}+t_{n}\right)\right) .
\end{array}
$$

Instead of the nilpotent generators $x_{i}$ of $B_{n}=F\left[x_{1}, \cdots, x_{n}\right]$ used in previous sections, we use at this point generators $z_{i}=1+x_{i}(i=1, \cdots, n)$. We have $z_{i}^{p}=1$, and every element of $B_{n}$ may be written uniquely in the form

$$
f=\sum_{s_{i} \in F_{p}} \alpha_{s_{1}} \cdots s_{n} z_{1}^{s_{1}} \cdots \cdots z_{n}^{s_{n}}, \quad \quad \alpha_{s_{1}} \cdots s_{n} \in F .
$$

Let $A$ in $K$ be of dimension $p^{n}$ so that $A^{+}=B_{n}$. Then (24) implies that multiplication in $A^{-}$is defined by

$$
[f, g]=\sum_{i, j} \frac{\partial f}{\partial z_{i}} \cdot \frac{\partial g}{\partial z_{j}} \cdot 2 c_{i j}
$$


since $\partial f / \partial z_{i}=\partial f / \partial x_{i}$. Equivalently, multiplication in $A^{-}$is defined by

$$
\begin{aligned}
& {\left[z_{1}^{s_{1}} \cdots \cdots z_{n}^{s_{n}}, z_{1}^{t_{1}} \cdots \cdots z_{n}^{t_{n}}\right]} \\
& \quad=\sum_{i, j=1}^{n} s_{i} t_{j} z_{1}^{s_{1}+t_{1}} \cdots z_{i}^{s_{i}+t_{i}-1} \cdots \cdots z_{j}^{s_{j}+t_{j}-1} \cdots \cdots z_{n}^{s_{n}+t_{n}} \cdot 2 c_{i j} .
\end{aligned}
$$

Let

$$
c_{i j}=0 \quad \text { unless } q_{k-1}+1 \leqq i, j \leqq q_{k} \text { for some } k(0 \leqq k \leqq m),
$$

$$
\begin{array}{ll}
2 c_{i j}=\alpha_{i j} z_{i} \cdot z_{j} & \text { for } 1 \leqq i, j \leqq n_{0}, \\
2 c_{i j}=\alpha_{i j} z_{i} \cdot z_{j} \cdot\left(z_{q_{k-1}+1} \cdots z_{q_{k}}\right)^{-1} & \quad \text { for } q_{k-1}+1 \leqq i, j \leqq q_{k}
\end{array}
$$

For typographical reasons we write $\left\{s_{1}, \cdots, s_{n}\right\}$ for $z_{1}^{s_{1}} \cdots \cdots z_{n}^{s_{n}}$. Then (45) and (46) imply

$$
\begin{array}{r}
{\left[\left\{s_{1}, \cdots, s_{n}\right\},\left\{t_{1}, \cdots, t_{n}\right\}\right]=\sum_{i, j=1}^{n_{0}} s_{i} t_{j} \alpha_{i j}\left\{s_{1}+t_{1}, \cdots, s_{n}+t_{n}\right\}} \\
+\sum_{k=1}^{n}\left(\sum _ { i , j = q _ { k - 1 } + 1 } ^ { q _ { k } } s _ { i } t _ { j } \alpha _ { i j } \left\{s_{1}+t_{1}, \cdots, s_{q_{k-1}}+t_{q_{k-1}}, s_{q_{k-1}+1}+t_{q_{k-1}+1}-1, \cdots\right.\right. \\
\left.\left.s_{q_{k}}+t_{q_{k}}-1, s_{q_{k+1}}+t_{q_{k}+1}, \cdots, s_{n}+t_{n}\right\}\right) .
\end{array}
$$

That is, $L \cong A^{-}$by (43) and (47), and $L / F u_{0} \cong A^{-} / F 1$.

In order to complete the proof of the theorem, we shall require the following

Lemma 2. Let $A$ be a noncommutative Jordan algebra such that $A^{+}$is associative. If $[f, g]=0$ for every $g \in A$, then $f \cdot A(=f A=A f)$ is an ideal of $A$. Thus, if $A$ is any simple nodal noncommutative Jordan algebra, $[f, g]=0$ for every $g \in A$ if and only if $f \in F 1$.

Proof. Clearly $f g=g f=f \cdot g$ for every $g \in A$. Then $[6,(4)]$ implies $(f \cdot g) h$ $=-(g \cdot h) f+(g h) \cdot f+(g f) \cdot h=-f \cdot g \cdot h+f \cdot(g h)+f \cdot g \cdot h=f \cdot(g h) \in f \cdot A$. If $A=F 1+N$ is simple, then either $f \cdot A=0$, implying $f=0$, or $f \cdot A=A$. In the latter case $f=\alpha 1+z, \alpha \neq 0, z \in N$. But then $[z, g]=[f-\alpha 1, g]=0$ for every $g$, implying $z=0, f=\alpha 1 \in F 1$.

We return to the proof of Theorem 7. $A$ is in $K$, since at least one of the $\alpha_{i j}$ in (46) is not zero [2, Theorem 2]. If $G=G_{0}$, then $L_{0}=L(G, \delta, f)=L / F u_{0}$ has dimension $p^{n}-1$. If $G \neq G_{0}$, then $L(G, \delta, f)=\left(L / F u_{0}\right)^{\prime}$ has dimension $p^{n}-2$. We shall show in both cases that $A$ is simple since $L(G, \delta, f)$ is. If $A$ is not simple, then $A$ has a nonzero ideal $B \subseteq N$. Since $N$ is not an ideal of $A$,

$$
1 \leqq \operatorname{dim} B \leqq p^{n}-2 .
$$

$B^{-}$is an ideal of $A^{-}$, and either $F 1 \cap B^{-}=F 1$, implying $1 \in B, B=A$, a con- 
tradiction, or $F 1 \cap B^{-}=0$. Hence $C^{-}=F 1 \oplus B^{-}$is an ideal of $A^{-}$. In case $G=G_{0}$, then $A^{-} / F 1 \cong L(G, \delta, f)$ is simple, so the kernel $C^{-} / F 1$ of the natural homomorphism of $A^{-} / F 1$ onto $A^{-} / C^{-}$is either 0 or all of $A^{-} / F 1$. That is, either $B^{-}=0$ or $\operatorname{dim} B^{-}=p^{n}-1$, contradicting (48) in either event. In case $G \neq G_{0}, L / F u_{0}=L(G, \delta, f)+F v$ contains an ideal $M$ corresponding to $C^{-} / F 1$ in $A^{-} / F 1$. Clearly $M \cong B^{-}$. Then $M \cap L(G, \delta, f)$ is an ideal of the simple algebra $L(G, \delta, f)$. In view of (48), it follows that either (i) $M=L(G, \delta, f)$, or (ii) $L / F u_{0}=L(G, \delta, f) \oplus M$ where $\operatorname{dim} B=\operatorname{dim} M=1$. In case (i), $L / F u_{0}$ $=M+F v$. Correspondingly, $A^{-} / F 1=C^{-} / F 1+F \bar{z}$ where $z$ may be taken to be in $N$. Then $A=C+F z=F 1+B+F z, N=B+F z$. Now $B$ an ideal of $A$ implies $N N=(B+F z)(B+F z) \subseteq B+F z^{2} \subseteq N$, a contradiction, since $A$ is a nodal algebra. There remains the possibility (ii), $L / F u_{0}=\left(L / F u_{0}\right)^{\prime} \oplus M$. Correspondingly, $\left(A^{-} / F 1\right)=\left(A^{-} / F 1\right)^{\prime} \oplus F \bar{w}$ where $w$ may be taken in $B$. Then $B=F w$. Since $B^{+}$is a 1 -dimensional ideal in $A^{+}=F\left[x_{1}, \cdots, x_{n}\right]$, we have $w=\sigma x_{1}^{p-1} \cdots x_{1}^{p-1}$ for $\sigma \neq 0 \in F$. Write $c_{i j}=\alpha_{i j} 1+z_{i j}$ in (24), $\alpha_{i j} \in F, z_{i j} \in N$. There exist $i_{0}, j_{0}$ such that $\alpha_{i_{0} j_{0}} \neq 0$. Then $\left[x_{i_{0}}, x_{1}^{p-1} \cdots \cdots x_{n}^{p-1}\right] \in B$ implies

$$
\begin{aligned}
0 & \equiv\left[x_{i_{0}}, x_{1}^{p-1} \cdots \cdots x_{n}^{p-1}\right] \equiv(p-1) \sum_{j}^{p-1} \cdots \cdots x_{j}^{p-2} \cdots \cdots x_{n}^{p-1} \cdot 2 c_{i_{0} j} \\
& \equiv 2(p-1) \sum_{i} \alpha_{i_{0} j} x_{1}^{p-1} \cdots \cdots x_{j}^{p-2} \cdots x_{n}^{p-1} \bmod B=F x_{1}^{p-1} \cdots \cdots x_{n}^{p-1},
\end{aligned}
$$

implying $\alpha_{i_{0} j_{0}}=0$, a contradiction. That is, $A$ must be simple if $L(G, \delta, f)$ is. That ad $A$ is isomorphic to $A^{-} / F 1$ follows directly from Lemma 2.

In $\$ 2$ we referred to the proof above for justification of the statement that any $A$ defined by a nondegenerate form $\phi$ is simple. This follows from the fact that $V_{r}=L(G, \delta, f)$ where $G_{0}=0, m=r$, and $G_{k}$ is of dimension 2 over $F_{p}$ for $k=1, \cdots, r\left[2\right.$, Lemma 3]. For then the $c_{i j}$ defined by (46) are all in $F 1$. In the proof of Theorem 6 we relied on (46) for the case $n=n_{0}=2$. In that instance $2 c=2 c_{12}=\alpha_{12} z_{1} \cdot z_{2}=\alpha_{12}\left(1+x_{1}\right) \cdot\left(1+x_{2}\right)$ with $\alpha_{12} \neq 0$.

We have not computed the derivations of the algebras $A$ in Theorem 7 . Instead we conclude with the following result which generalizes (26) in the direction of (14).

THEOREM 8. Let $A$ be in $K$ so that multiplication is defined by (1). If $A^{-}$is a Lie algebra, then the mappings $D$ defined by (4) with

$$
a_{i}=\sum_{j=1}^{n}\left(\frac{\partial g}{\partial x_{j}}+\alpha_{j} x_{j}^{p-1}\right) \cdot c_{i j}, \quad i=1, \cdots, n,
$$

for any $g \in A$ and any $\alpha_{j} \in F(j=1, \cdots, n)$, are derivations of $A$.

Since ad $g / 2$ in (25) is a derivation of $A$, it is sufficient to verify that $D$ in (4) is a derivation in case

$$
a_{i}=\sum_{k=1}^{n} \alpha_{k} x_{k}^{p-1} \cdot c_{i k}, \quad \alpha_{k} \in F .
$$


Now $D$ is a derivation of $A$ in case (5) with $k$ replaced by $t$ is satisfied. But (50) implies

$$
\begin{aligned}
\sum_{t=1}^{n} & \left(\frac{\partial c_{i j}}{\partial x_{t}} \cdot a_{t}+\frac{\partial a_{i}}{\partial x_{t}} \cdot c_{j t}+\frac{\partial a_{j}}{\partial x_{t}} \cdot c_{t i}\right) \\
= & \sum_{k, t}\left(\frac{\partial c_{i j}}{\partial x_{t}} \cdot \alpha_{k} x_{k}^{p-1} \cdot c_{t k}+\alpha_{k} \frac{\partial\left(x_{k}^{p-1} \cdot c_{i k}\right)}{\partial x_{t}} \cdot c_{j t}+\alpha_{k} \frac{\partial\left(x_{k}^{p-1} \cdot c_{j k}\right)}{\partial x_{t}} \cdot c_{t i}\right) \\
= & \sum_{k} \alpha_{k} x_{k}^{p-1} \cdot\left\{\sum_{t}\left(\frac{\partial c_{i j}}{\partial x_{t}} \cdot c_{t k}+\frac{\partial c_{i k}}{\partial x_{t}} \cdot c_{j t}+\frac{\partial c_{j k}}{\partial x_{t}} \cdot c_{t i}\right)\right\} \\
& +\sum_{k} \alpha_{k} x_{k}^{p-2} \cdot\left(c_{i k} \cdot c_{j k}+c_{j k} \cdot c_{k i}\right) \\
& =0
\end{aligned}
$$

by (27).

\section{REFERENCES}

1. A. A. Albert and M. S. Frank, Simple Lie algebras of characteristic p, Rend. Sem. Mat. Torino vol. 14 (1954-1955) pp. 117-139.

2. Richard Block, New simple Lie algebras of prime characteristic, Trans. Amer. Math. Soc. vol. 89 (1958) pp. 421-449.

3. M. S. Frank, A new class of simple Lie algebras, Proc. Nat. Acad. Sci. U.S.A. vol. 40 (1954) pp. 713-719.

4. Nathan Jacobson, Classes of restricted Lie algebras of characteristic p. II, Duke Math. J. vol. 10 (1943) pp. 107-121.

5. L. A. Kokoris, Some nodal noncommutative Jordan algebras, Proc. Amer. Math. Soc. vol. 9 (1958) pp. 164-166.

6. - Simple nodal noncommutative Jordan algebras, Proc. Amer. Math. Soc. vol. 9 (1958) pp. 652-654.

7. - Nodal noncommutative Jordan algebras, to appear in Canad. J. Math.

8. D. G. Northcott, Ideal theory, Cambridge, 1953.

9. R. D. Schafer, On noncommutative Jordan algebras, Proc. Amer. Math. Soc. vol. 9 (1958) pp. 110-117.

INSTITUTE FOR ADVANCED STUdy,

Princeton, New Jersey

UNIVERSITY OF CONNECTICUT, STORRS, CONNECTICUT 